\title{
MULTILAYERED STRUCTURES
}

\author{
M.K. Sanyal, A. Datta and S. Hazra \\ Surface Physics Division, Saha Institute of Nuclear Physics 1/AF Bidhannagar, \\ Kolkata 700064
}

\section{INTRODUCTION}

Artificially structured multilayered materials are formed by depositing stacks of nanometer sized layers of two or more materials. In the growth direction, apart from the microscopic lattice constants of deposited materials, presence of "interfaces" between two layers defines a "superlattice", i.e., an additional one dimensional (1D) periodicity, which modifies considerably the physical properties of a multilayered structure. In fact, one can tune electrical, magnetic, optical and mechanical properties of these materials by controlling the shape of this 1D periodicity and the structure of the surfaces and interfaces. This is one of the most active research fields in physics, not only because of its wide ranging technological applications but also due to the possibility of testing existing and creating new ideas regarding the fundamentals of material science. Advent of excellent techniques for growing these multilayered structures and recent advances in methods for characterization of the interfacial structure and nature of interfacial roughness of these materials with angstrom accuracy have made this area of research very active in all the advanced countries. In our country various research institutes like, Saha Institute of Nuclear Physics, Tata Institute of Fundamental Research, Bhabha Atomic Research Centre, Indian Institute of Science, Indian Institutes of Technology, Institute of Physics and Inter University Consortium for DAE facilities have initiated research programme in this emerging area [1].

The overwhelming majority of research studies in the subjects of multilayers are concerning semiconducting materials. This research has applications in the area of high-speed devices and in optical communications. This activity has also given us exciting fundamental knowledge regarding low-dimensional systems. Many semiconductor multilayered struc- 
tures have been produced and their complexity is increasing [2]. In semiconductors the directional nature of chemical bonds results in the formation of extremely high quality superlattices. In metals, however, the bonding is less directional and it is more difficult to achieve comparable perfection. Nevertheless state-of-the-art advanced crystal growth methods like molecular beam epitaxy (MBE) have now made possible the sequential monolayer by monolayer deposition not only of semiconductors but also for metals and insulators, and, practically any combinations thereof. Compositionally modulated multilayers have found a wide interest both in basic research as well as in some areas of electronic and opto-electronic devices, $\mathrm{x}$-ray mirror structures, magnetic recording devices, hard surface coatings etc. A recent experimental advance, namely strained-layer epitaxy has enabled us to tailor the band-gap almost at will and the production of such artificially structured materials may be called "band-gap engineering". The correct choice of materials permits the spatial variation of the band structure in the growth direction (perpendicular to the film surface). Moreover presence of superlattice period helps to convert materials like silicon having indirect band-gap to a material with direct band-gap.

Atomically engineered metallic multilayer materials have attracted tremendous interest [3] because of their potential in emerging technologies as well as in basic research. From application point of view these materials, having one magnetic constituent, are promising candidates for reading heads in the next generation of high-density data storage systems and future magneto-optic recording media. There are some unresolved issues in basic research such as mechanism of interdiffusion and alloy formation at the metallic multilayer interfaces and the role of the interfacial structure in the giant magneto-resistance (GMR) property which magnetic multilayers possess. These materials are formed by depositing alternatively ferromagnetic and non-magnetic layers each having thickness around $10-20 \AA$. When an external magnetic field is applied, the moments of these magnetic layers, which couple antiferromagnetically with each other normally, align ferromagnetically and due to this change in magnetic structure, the electrical resistance of these systems can decrease significantly [3]. From the recent publications [3-5], it is becoming increasingly clear that interfacial structures play an important role in the physical properties of these materials. It is been observed that in magnetic multilayers, the GMR with current perpendicular to the layers is dependent on both multilayer structure and the interface roughness. But the GMR with current parallel to the layers is mainly due to the interface roughness.

Multilayered organic and metal-organic films are being studied actively to form model systems for biophysics application and to understand the self-assembling mechanism observed in physics and biology. Using Langmuir-Blodgett (LB) technique ordered multilayer films of these materials, can be deposited by a relatively unsophisticated technique of repeated dipping of a solid substrate through a Langmuir monolayer generally formed by spreading 
amphillic molecules on water surface [6,7]. Although it was known that these LB films have well-ordered structure along the growth direction, the growth mechanism is not very well understood [8]. Results of some recent experiments [9] on LB films suggest that this is almost ideal system for studying melting of two-dimensional solids, which is expected to be a continuous transition [10] as opposed to melting of conventional three-dimensional solids.

Surface scientists have traditionally used experimental probes that have small penetration depth, such as electrons to study the structure of atoms at the surfaces and interfaces (which are invariably present with bulk atoms!). However, due to strong interactions of electrons at the surface, multiple scattering take place and as a result quantitative structure determination using only electron diffraction becomes problematic. By using x-rays and neutrons in grazing incidence, the scattering can be made surface sensitive and these radiations are increasingly being used to probe structure of surfaces and interfaces [11]. Scanning Probe Microscopy (SPM) is another technique, which is finding wide application in surface characterization of various materials. Real space surface images obtained from this technique provide information complementary to the statistically averaged reciprocal space information obtained from scattering techniques [1]. In this article we shall discuss the present status and the possible future projections of the above-mentioned multilayered structures with emphasis on growth and structural aspect of these systems. X-ray scattering and SPM techniques, owing to the near atomic resolution, play major role in the development of these materials. In the subsequent sections, we shall also discuss the basics of these techniques to develop better understanding about the present status of multilayered structures.

\section{CHARACTERIZATION TECHNIQUES}

\section{X-Ray Scattering}

Structural studies of multilayers using grazing incidence x-ray scattering (GIXS) techniques are becoming a very prominent field of research [11-13] due to the availability of intense rotating anode sources and synchrotron sources. Moreover these techniques can probe buried interfaces of multilayered structures non-destructively. In these studies x-ray scattering intensities are monitored as a function of various grazing angles of incidence and as this angle increases scattered radiation provides us information regarding buried interfaces. Xray sources of high brightness are needed for gathering sufficient scattered $\mathrm{x}$-ray intensity to study structures of surfaces and interfaces, as the number of atoms at surface and interfaces is orders of magnitude smaller than that in the bulk. 


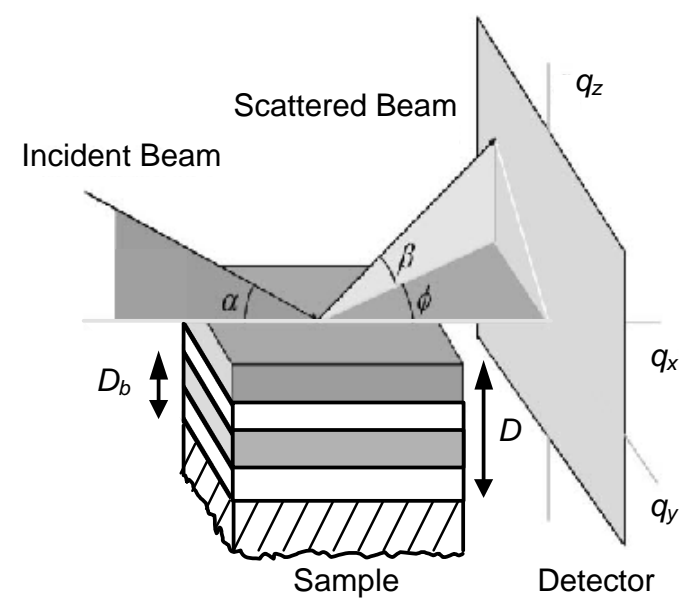

Figure 1: Schematic of the scattering geometry used to perform x-ray measurements (refer text for details). A multilayer film (of total thickness $D$ ) having two bilayers each of thickness $D_{\mathrm{b}}$, on a substrate is shown. Results of analysis of similar bilayers are given in the text (refer Figure 2).

Three different measurements are used in GIXS to give different information regarding the interfacial structures. These are specular reflectivity, diffuse scattering and grazing incidence diffraction (GID). Schematic of the scattering geometry used to perform different $x$ ray measurements is shown in Figure 1. The surface of the sample is located in the $x-y$ plane and the incident $\mathrm{x}$-ray beam is in the $x-z$ plane. Here $\alpha$ is the incident angle with the $x-y$ plane and $\beta$ and $\phi$ are the exit angles with the $x-y$ and $x-z$ planes, respectively. The components of the wave vector transfer, $\boldsymbol{q}\left(q_{x}, q_{y}, q_{z}\right)$, corresponding to the incident wavelength $\lambda$ are $q_{x}=(2 \pi / \lambda)(\cos \beta \cos \phi-\cos \alpha), q_{y}=(2 \pi / \lambda)(\cos \beta \sin \phi), q_{z}=(2 \pi / \lambda)(\sin \alpha+\sin$ $\beta)$. For reflectivity and diffuse scattering measurements, $\phi=0$ yielding $q_{x}=(2 \pi / \lambda)(\cos \beta-$ $\cos \alpha)$ and $q_{y}=0$. In x-ray specular reflectivity experiments, a well collimated monochromatized incident beam strikes at an angle $\alpha$ (starting from few milliradians) and the scattered intensity is recorded using a detector placed after a tight slit in the plane of incidence also at an angle $\beta(=\alpha)$. In reflectivity measurements, $\alpha=\beta=\theta$, so that $q_{x}=0$ and $q_{z}=$ $(4 \pi / \lambda)(\sin \theta)$, the scattering vector $\boldsymbol{q}$ is perpendicular to the surface (specular direction), providing information about the mean electron density as a function of depth $(z)$. To have better understanding of the correlation in an interfaces and between interfaces, we need a technique which is sensitive to in-plane morphology and grazing incidence $\mathrm{x}$-ray diffuse scattering directly provides us this information [14]. Generally two types of diffuse data are collected to extract morphological information through height-height correlation at one in- 
terface and between interfaces in a multilayer. For transverse diffuse scattering measurements, the scattering angle $\alpha+\beta=2 \theta$ is maintained at a fixed value, while $\alpha$ and $\beta$ are scanned. The longitudinal diffuse data are taken along the specular direction by keeping offset between $\alpha$ and $\beta$ suitably in this scan so that $q_{x} \neq 0$. The collected diffuse data are then plotted with proper normalization so that specular, transverse and longitudinal data are all self-consistent. In GID, one obtains in reciprocal space, rods of intensities, known as the crystal truncation rods (CTR), corresponding to a surface structure [15] instead of spots obtained for 3D structures. Once the intensities of these rods are measured, methods of analysis for GID data are similar to that used in conventional x-ray crystallography measurements. In these measurements the x-ray beam is incident at a fixed angle $\alpha$, just below the critical angle, $\alpha_{c}$ of the film material and the scattered intensity is measured as a function of $\beta$ and $\phi$.

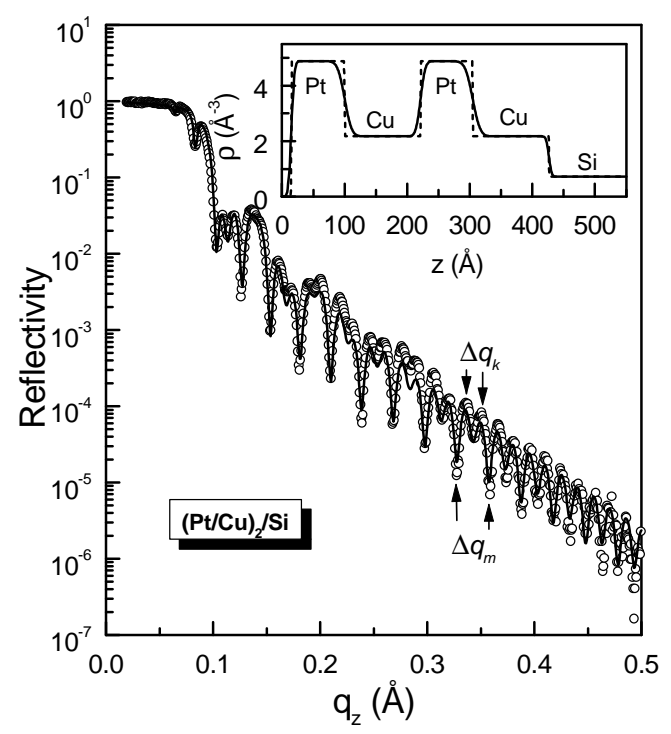

Figure 2: Experimental (symbol) and fitted (line) x-ray reflectivity profile for a multilayered film. Corresponding electron density profiles convoluted with error function (solid line) and without convolution (dotted line) in the inset. Arrows indicate the separation between two Kiessig fringes $\left(\Delta q_{k}\right)$ and between two modulations $\left(\Delta q_{m}\right)$.

\section{Interpretation of Reflectivity Data}

X-ray reflectivity curve of a typical multilayered thin film is shown in Figure 2. The interference (Kiessig) fringes observed in the reflectivity curve are due to total film thickness. The modulation in the fringes is due to the presence of bilayer period. If $\Delta q_{k}$ and 
The modulation in the fringes is due to the presence of bilayer period. If $\Delta q_{k}$ and $\Delta q_{m}$ be the separation between two consecutive Kiessig fringes and between two consecutive modulations respectively, then the total film thickness $(D)$ and the bilayer separation $\left(D_{\mathrm{b}}\right)$, refer Figure 1, can be estimated as $D=\pi / \Delta q_{k}$ and $D_{\mathrm{b}}=\pi / \Delta q_{m}$.

One needs to carry out detailed analysis of the reflectivity data to get accurate information regarding the bilayer thickness, interfacial widths and electron densities of the individual layers in a multilayered thin film [16-18]. Since x-ray reflectivity profile is sensitive to this electron density distribution of the system, it is of usual practice to divide the film into slabs having different electron densities for analysis. The $x-y$ average electron density profile (EDP) as a function of depth, $\rho(z)$ can then be written as

$$
\rho(z)=\sum_{1}^{n} \Delta \rho_{i} f\left(z_{i}, \sigma_{i}\right)
$$

where $\Delta \rho_{i}$ is the change in electron density at $i$ th interface located at a position $z_{i}$ and $f$ is an error function given by

$$
\begin{aligned}
f\left(z_{i}, \sigma_{i}\right) & =f\left(z-z_{i}, \sigma_{i}\right) \\
& =\sigma_{i}^{-1}(2 \pi)^{-1 / 2} \int_{-\infty}^{z-z_{l}} \exp \left(-t^{2} / 2 \sigma_{i}^{2}\right) d t
\end{aligned}
$$

where $\sigma_{i}$ is the roughness of the $i$ th interface which is a parameter for the estimation of interfacial width. For the analysis of the present $(\mathrm{Pt} / \mathrm{Cu})_{2} / \mathrm{Si}$ multilayered system, we divide the film into 4 slabs of equal thickness plus substrate of infinite thickness (refer Figure1). As a starting guess, the total thickness of the film was estimated from the Kiessig fringes and the individual layer thicknesses were assumed to be one-fourth of the total. The electron densities of these 4 slabs were then estimated considering alternatively $\mathrm{Pt}$ and $\mathrm{Cu}$ layers. The bulk value of $\rho$ for $\mathrm{Pt}, \mathrm{Cu}$ and $\mathrm{Si}$ are 4.886, 2.289 and 0.714 , respectively which yields $\Delta \rho_{1}=4.886, \Delta \rho_{2}=-2.597, \Delta \rho_{3}=2.597, \Delta \rho_{4}=-2.597, \Delta \rho_{5}=-1.575$. The initial estimate of $\sigma$ for all the interfaces was $5 \AA$. It can be noted that the error functions of Eq.(2) come as Debye-Waller like functions for the reflectance of each interface in reciprocal space

$$
r^{u, v}=r_{F}^{u, v} \exp \left(-\frac{q_{z}^{2} \sigma_{u}^{2}}{2}\right)
$$


The reflectivity of a multilayer, calculated [12,13] using these reflectances for the interfaces, was then fitted to the experimental data using thickness, $\rho$ and $\sigma$ of each layer as fitting parameters. Best-fit curve thus obtained is shown in Figure 2 along with EDP in the inset. The locations of different materials in the film are also indicated in the EDP. Different parameters obtained from the analysis are the total film thickness $412( \pm 2) \AA$, thickness of the individual layers from the top of the film $86,101,83,122 \AA$ corresponding electron density $4.85,2.17,4.85,2.17( \pm 0.05) \AA^{-3}$ respectively. The roughness from the top of the film obtained are 3.9, 8.9, 6.0, 9.5, 3.6 $\AA$. Analysis shows that the value of $\rho$ for the Pt layers is almost same that of bulk $\mathrm{Pt}$ and for the $\mathrm{Cu}$ layers it is $\sim 5 \%$ less than that of bulk $\mathrm{Cu}$. It also shows that the interface of heavy metal $\mathrm{Pt}$ deposited on $\mathrm{Cu}$ is broader than the interface of $\mathrm{Cu}$ deposited on Pt. The above analysis of the thin film indicates the formation of a near perfect multilayered system. However, it is interesting that slight deviations from perfection can also be estimated from the analysis and many sensitive analysis schemes have been developed for this purpose [17-19].

\section{Analysis of Diffuse Scattering Data}

Off-specular diffuse scattering studies probe in-plane height-height correlation of a thin film [14]. These studies also provide us information regarding the conformality of interfaces in a multilayer, which measures the similarity between interfaces [20]. The scattering process is generally treated in Born approximation to estimate the effect of in-plane correlation in the measured diffuse scattering profiles. In this approximation one can write $S(\boldsymbol{q})$, which is the Fourier transform of the density-density correlation function, as

$$
S(\vec{q})=\left|\int d r \rho_{e l}(\vec{r}) \exp (-\overrightarrow{i q} \cdot \vec{r})\right|^{2}
$$

Here both $\vec{q}$ and $\vec{r}$ are three-dimensional vectors and $\rho_{e l}$ is the electron density distribution. In most cases, it is possible to separate out the specular component from the diffuse intensity which arises due to correlation of heights as a function of lateral separation $R$ [14]. Assuming that these heights $z\left(R \equiv \sqrt{x^{2}+y^{2}}\right)$ are Gaussian random variables, we get

$$
g(R)=\left\langle[z(0)-z(R)]^{2}\right\rangle=2 \sigma_{G}^{2}+2\langle z(0) z(R)\rangle
$$


Here $\sigma_{G}$ is the root mean squared roughness parameter and $C(R) \equiv\langle z(0) z(R)\rangle$ is the heightheight correlation that characterizes a system or an interface. For multilayers, i.e. a system having multiple interfaces, one can write a simplified expression for scattering intensity by assuming complete correlation in the interfaces [21,22]

$$
I\left(q_{R}, q_{z}\right)=\frac{I_{0}}{q_{z}^{2}} \frac{b^{2}}{k \sin \alpha \sin \beta} \sum_{i, j} \Delta \rho_{i} \rho_{j} \exp \left(-i q_{z}\left(z_{i}-z_{j}\right)\right) \exp \left(-i q_{z} \sigma_{G}^{2}\right) F_{i, j}\left(q_{R}\right)
$$

In Eq. (6) $\Delta \rho_{i}$ represents difference between electron densities of the medium above and below the $i$ th interface, $b$ is the classical electron radius and the factor $F_{i, j}\left(q_{R}\right)$ represents a convolution with the instrumental resolution function in $q_{x}$ and $q_{y}$

$$
F_{i, j}\left(q_{R}\right)=\iint d x d y \exp \left(q_{z}^{2} C_{i j}(R)\right) \exp \left(i q_{R} R\right)
$$

Here $C_{i j}(R)$ is the height-height correlation for multilayered system [22]. By splitting Eq. (6) into two parts, one of which yields the specular reflectivity and the other the diffuse scattering intensity [20], the expression for diffuse component becomes [19]

$$
I\left(q_{x}, q_{z}\right)=I_{0} R\left(q_{z}\right) \frac{\lambda q_{z}}{4 \pi \sin \alpha} \int\left[\exp \left(q_{z}^{2} C(x)\right)-1\right] \exp \left(-i q_{x} x\right) d x
$$

where $I_{0}$ is the direct beam intensity and $R\left(q_{z}\right)$ is the reflectivity. In the above expression we assume that all the interfaces are conformal and resolution out of the scattering plane is relaxed in such a way that integration over $q_{y}$ has been performed during data collection. Analysis schemes based on distorted wave Born approximation have been developed to calculate these intensity profiles near critical angles and for systems not having complete conformality [12-14]. We shall present here the results of a conformal system analyzed using Eq. (8) for simplicity. The height-height correlation function at lateral separation $R$ between two conformal, self-affine and rough interfaces $i$ and $j$ used here is [14,19]

$$
C_{i j}(R)=\left\langle z_{i}(0) z_{j}(R)\right\rangle=\sigma^{2} \exp \left[-\left(\frac{R}{\xi}\right)^{2 h}\right]
$$




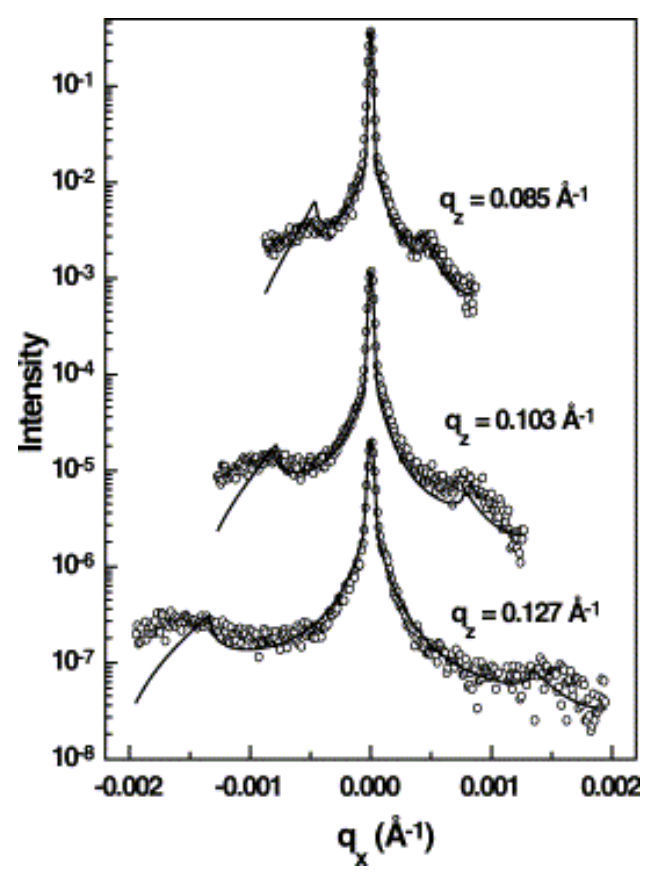

Figure 3: Transverse diffuse scattering intensity (open circles) as a function of $q_{x}$ for three different values of $q_{z}$ for a quantum-well sample along with fit (solid line).

In Figure3 we have shown the result of analysis of the transverse diffuse data taken at three different $q_{z}$ values for a quantum well structure [19]. We obtained the values of $\sigma$, $\xi$, and $h$ as $5.5 \AA 10000 \AA$, and 0.45 , respectively. The same set of parameters was used to selfconsistently calculate the longitudinal diffuse scattering profile [Figure 4. Top panel]. This profile follows the specular reflectivity profile (top) closely, indicating conformality. It is known that EDP obtained from the x-ray specular reflectivity study is actually a convolution of compositional and interfacial roughness profiles. As the roughness here is conformal, one can obtain compositional profile by deconvoluting the $\rho^{\prime}(\mathrm{z})$ with the Gaussian having $\sigma \sim 5.5 \AA$, corresponding to the interfacial roughness. This deconvolution can be performed in Fourier space by utilizing the fact that the Fourier transform of the convolution of two functions is the product of the Fourier transforms of the functions. A Gaussianlike (with variance of $\sigma_{G}$ ) derivative profile of EDP is shown [Figure 4. Bottom panel] along with the roughness Gaussian $(\sigma \sim 5.5 \AA)$, obtained from diffuse scattering analysis, at both the interfaces of the quantum well. The values of $\sigma_{G}$, found by fitting Gaussian functions, were 12 and $9 \AA$ for the quantum-well interfaces with the cap layer and substrate, respectively. This indicates that the interfacial profile is dominated by interdiffusion and 
substrate-quantum- well interface is sharper than the quantum-well cap-layer interface. It is also interesting to note that we obtained high in-plane correlation length for this epitaxial system having predominant interdiffusion, as observed earlier. Deconvoluted compositional profile is not presented here be-cause the obtained profile is not much different from the EDP.
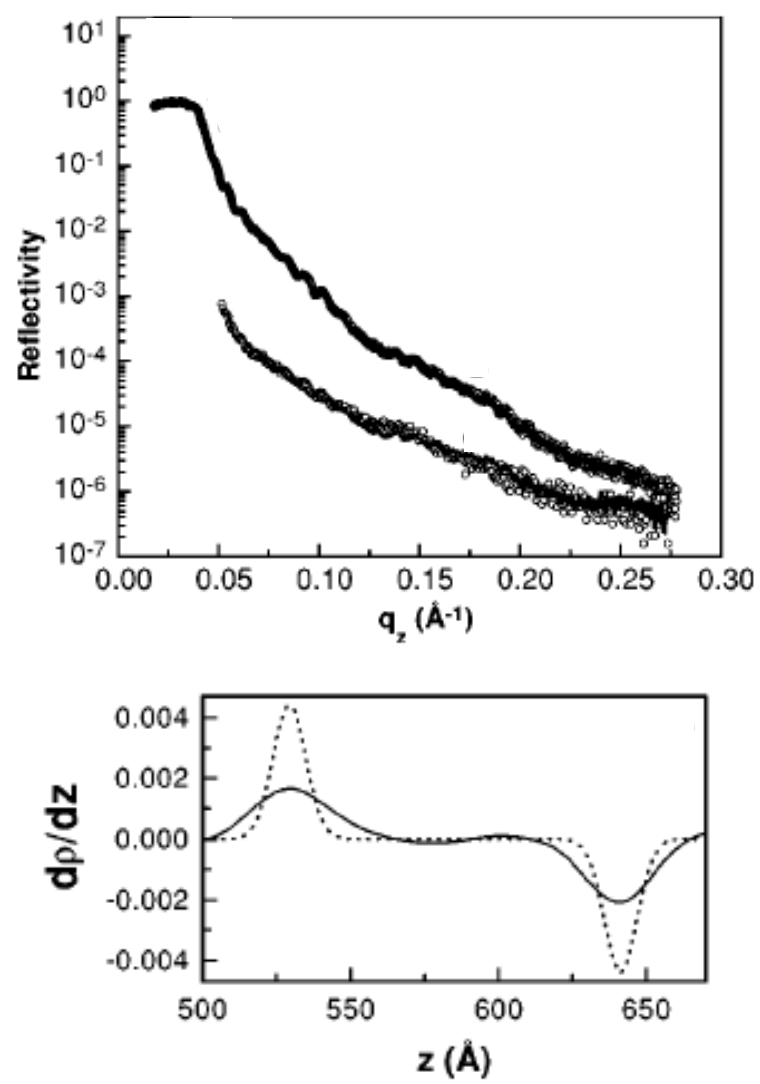

Figure 4: Top panel: Specular reflectivity (top) and longitudinal off-specular reflectivity (bottom) for quantum-well sample. Experimental data are shown by open circles and fitted curves by solid lines. Bottom panel: Derivative of final EDP (solid line) and Gaussian function with $\sigma=5.5 \AA$ (dotted line) corresponding to the conformal interfacial roughness in the quantum-well region.

For an epitaxial system, like this quantum well, one can extract more information regarding the interfacial structure by high resolution diffraction measurements. The extracted 
composition profile from reflectivity and diffuse scattering measurements can then be used to calculate the expected diffraction profile. In Figure 5 we have shown calculated and experimental (004) diffraction data. The lattice parameter of the quantum well obtained from the diffraction data is $5.71 \AA$, which was used along with the obtained composition profile to extract the elemental composition. The composition near the center of the quantum well was found to be $\operatorname{In}_{0.40} \mathrm{Ga}_{0.60} \mathrm{As}_{0.48} \mathrm{P}_{0.52}$, which is quite different from the desired composition $\left(\operatorname{In}_{0.33} \mathrm{Ga}_{0.67} \mathrm{As}\right)$. $A_{j}$ for the entire film was calculated from the obtained compositional profile and the values of $d_{j}$ used in the experimental data could be obtained from this calculation. The lattice strain profile at the interfaces for such epitaxial multilayer structure can be obtained by fitting the calculated profile with the measured

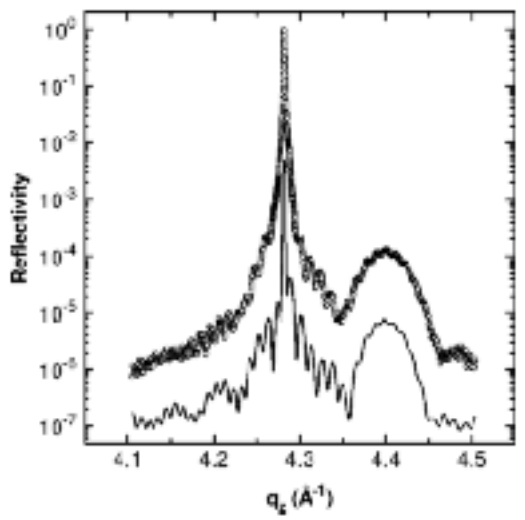

Figure 5: Measured (open circles) and calculated $(\times 0.1$, solid line $)$ reflectivity around the (004) Bragg peak as a function of $q_{z}$ for a quantum-well sample. diffraction data.

\section{Grazing Incidence Diffraction Technique}

Grazing Incidence Diffraction (GID) of x-rays from any interface requires a total external reflection of x-rays at the interface, i.e., when the incident angle $\alpha<\alpha_{c}$, where $\alpha_{c}$ is the critical angle between the two media. Under this condition, the refracted wave vector $\vec{k}^{\prime}$ is given by

$$
k_{x}^{\prime}=k \cos \alpha, k_{y}^{\prime}=0, \vec{k}_{z}=-i k \sqrt{\cos ^{2} \alpha-\cos ^{2} \alpha_{c}}
$$

The imaginary value of $k_{z}^{\prime}$ leads to a damping of the wave field (to $e^{-1}$ of its value at the interface) within a depth of $l$ below the interface given by

$$
l \equiv 1 /\left|\operatorname{Im} k_{z}^{\prime}\right| \cong \lambda / 2 \pi \sqrt{\alpha_{c}{ }^{2}-\alpha^{2}},
$$

for the x-ray wavelength $\lambda \approx 1 \AA, l$ ranges from $25 \AA$ to $100 \AA$ inversely as the electron density of the lower medium. Thus the incident $\mathrm{x}$-rays are confined within a depth of a few 
hundred A below the interfacial plane whereas they travel in the plane ( $x$ and $y$ directions) with a wavelength close to the free-space wavelength. This is the condition for GID that is exploited to study the structure of the interfacial region at atomic resolution. Using the Fresnel equations for electromagnetic waves and the above conditions we can construct the totally reflected $\mathrm{x}$-ray wave due to grazing incidence at the interface. This wave is now considered to scatter from the dielectric distributions at the individual atoms in this interfacial region and give rise to a diffraction pattern. The separation of the scattering process into these two steps is the basis of the Distorted Wave Born Approximation [23].

A particularly simple application of GID has been to determine the structures of Langmuir monolayers, i.e., monolayers of amphiphilic molecules at the air-water interface. These monolayers, the precursors of LB multilayered films on solid substrates, exhibit phase transitions with surface pressure, temperature, $\mathrm{pH}$ and ion content of the water. The transitions involve changes in two-dimensional lattice structure, tilt magnitude and direction of the molecules, and orientation of the molecular backbone planes. Here we present, as an example, a typical result from a systematic GID study of heneicosanoic acid monolayers. This is a fatty acid with a long hydrocarbon chain $\left(\mathrm{COOH}\left(\mathrm{CH}_{2}\right)_{19} \mathrm{CH}_{3}\right)$. The structures of different phases of these monolayers at close to zero surface pressure have been studied with two different divalent metal cations with close values of electronegativity (cadmium and zinc) in the subphase (water), and with variations in subphase $\mathrm{pH}$ and temperature [24]. In these experiments the $\mathrm{x}$-rays were made incident at a fixed angle $\alpha$ slightly lower than the critical angle for water and the scattered $x$-ray intensity was measured as a function of the in-plane and out-of-plane scattering angles $\phi$ and $\beta$. The expression for out-of-plane component of scattered wave vector $q_{z}$ has already been shown. The in-plane component is given as

$$
q_{x y}=\frac{2 \pi}{\lambda} \sqrt{\cos ^{2} \alpha+\cos ^{2} \beta-2 \cos \alpha \cos \beta \cos \phi}
$$

As Langmuir monolayers are powders in the plane the in-plane component of $\vec{q}\left(q_{x y}\right)$ cannot be decomposed further into $x$ and $y$ components. The total momentum transfer lies in a plane normal to the hydrocarbon chains and hence, if chains are tilted to the water surface some or all of the diffraction peaks will have maximum intensity at nonzero $q_{z}$.

Figure 6 (top) depicts the $q_{x y}$ scan of the monolayer at $9.2^{\circ} \mathrm{C}$ with subphase (having $10^{-4} \mathrm{M}$ $\mathrm{Cd}^{2+}$ ions) $\mathrm{pH} \sim 6.2$. The $q_{x y} / q_{z}$ contour plot of the scanned region is shown in Figure 6 (bottom). It shows an in-plane and an out-of-plane peak. By fitting the peaks in the out-of plane scans (vertical to the contours) to Gaussian and the peaks in the in-plane scans (horizontal to the contours) to Lorentzian functions the $q_{z}$ and $q_{x y}$ positions were obtained. The spacings 


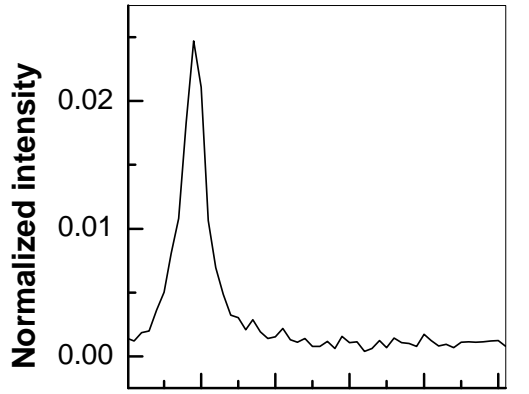

$\begin{array}{lllll}1.45 & 1.50 & 1.55 & 1.60 & 1.65\end{array}$

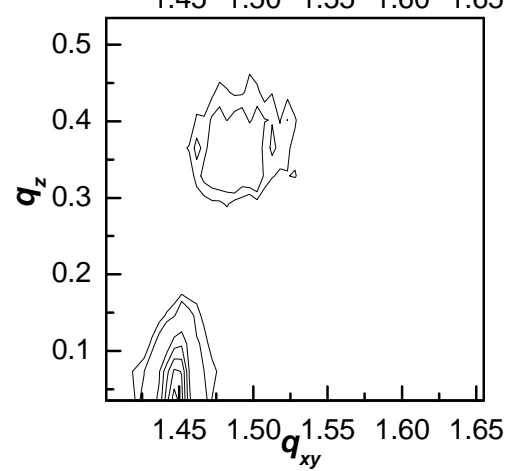

Figure 6: In-plane (top) and total (bottom) GID scans of heneicosanoic acid Langmuir monolayer. of the two-dimensional lattice contributing to a $q_{x y}$ peak is then given by

$$
q_{x y}^{h k}=\frac{2 \pi}{d_{h k}}
$$

The tilt angle $\tau$ and the azimuthal angle $\psi_{h k}$, with respect to the specific $[h k]$ direction are given in terms of $q_{z}$ and $q_{x y}$ peaks as

$$
q_{z}^{h k}=q_{x y}^{h k} \cos \psi_{h k} \tan \tau
$$

In absence of higher order peaks, the peak at lower $q_{x y}$ was tentatively assigned to degenerate reflections from (11) and (1 $\overline{1})$ planes and the higher $q_{x y}$ to reflection from (20) planes of a centred rectangular unit cell (with two molecules per unit cell). The parameters of this cell are: $a=4.98 \AA, b=8.62 \AA$. The hydrocarbon chains were found using Eq. (13) to be tilted towards a nearest neighbor by an angle of about $22.3^{\circ}$ from the vertical. In the analysis of LB and other multilayer films similar procedure is followed [15] and some examples will be given in next section.

\section{Scanning probe microscopy}

Soon after the commercialization of the tunneling phenomenon in form of Scanning Tunneling Microscope (STM), a series of techniques evolved to characterise the top surface morphology in real space starting from micron to angstrom resolution. These techniques come under the common family- Scanning Probe Microscopy (SPM), of which Atomic Force Microscopy (AFM) is widely used as it can practically probe any types of surfacesfrom metallic to non-metallic, from hard to soft ones. As the name suggests, STM operates by the mechanism of tunneling. Electrons tunnel from a small metallic tip held above the surface into the surface if the tip is biased negatively. The direction of tunneling current is reversed with the bias. The vertical resolution is achieved by the exponential dependence of the tunneling current on the tip-to-surface separation. A typical variation in current is an 
order of magnitude for every angstrom separation. An image in the lateral direction is achieved by scanning the tip across the surface. AFM appears to be in more general use since it no longer necessitates conducting tip and sample as STM does. In AFM the probing tip is attached to a cantilever which is deflected in response to the atomic forces such as van der Waal's forces between the probing tip and the sample [25].

Typical images as obtained by AFM and STM are shown in Figure 7. The Top-Left AFM image is of freshly cleaved mica surface showing the atomic arrangement. The Top-Right AFM image is of a thin film of silver. Island like morphology of the film is clear from this
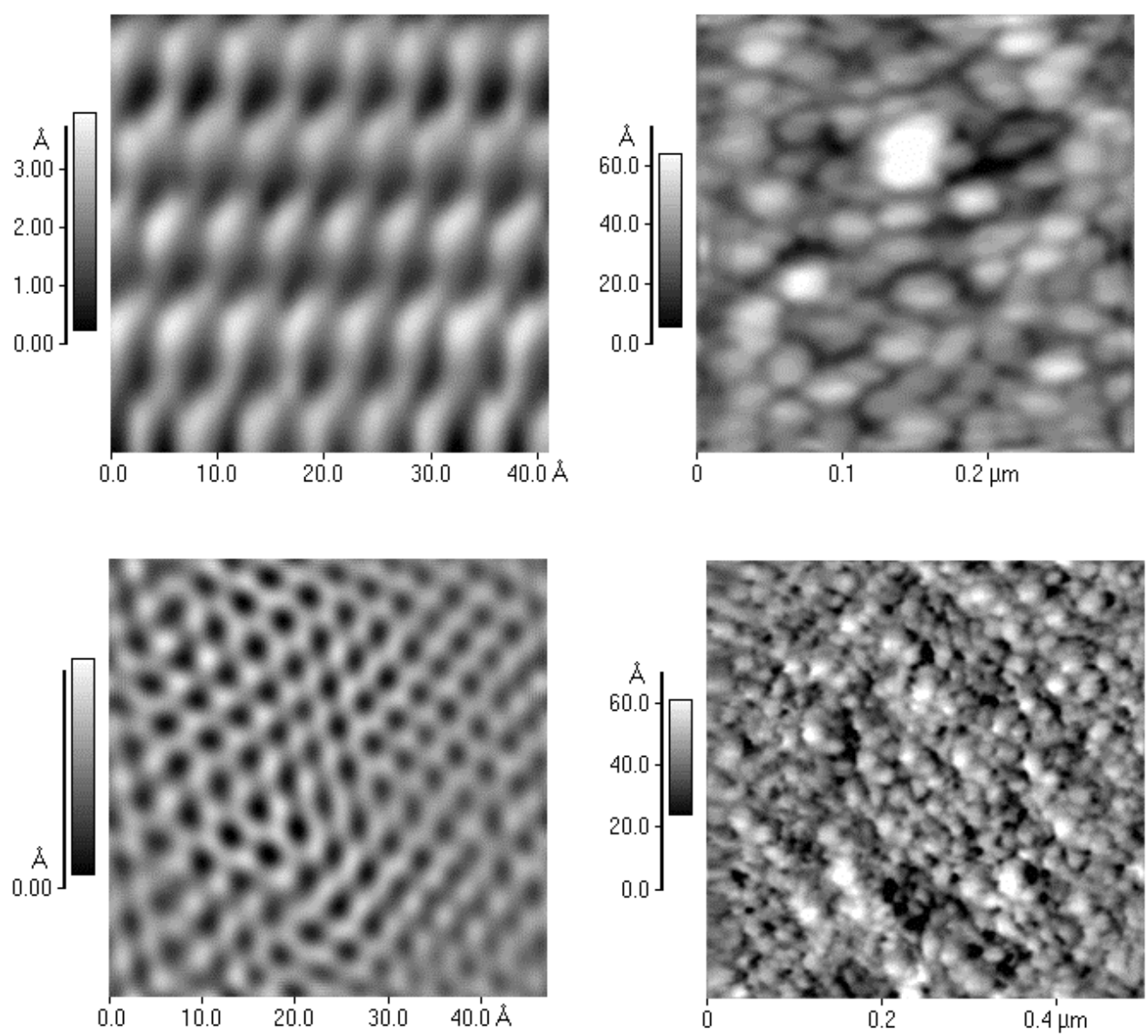

Figure 7: AFM (top) and STM (bottom) images in different resolutions. Left: atomic resolution, Right: morphology in micron dimension. 
image. The Bottom-Left STM image is of freshly cleaved graphite surface showing atomic arrangement. The Bottom-Right STM image is of a silver film.

Although SPM only gives near surface information, its proper use provides valuable information about multilayer structured systems. In-plane molecular arrangement of LB films and its monolayer or bilayer thickness can be very well determined using AFM [6]. Study of such LB films with different number of layers provides information about the layer by layer molecular arrangements in these films. AFM studies over different length scales are not only useful to map the morphology but also to estimate the defects and surface roughness. The scaling of roughness of each surface and its correlation with other interfaces provides valuable information regarding overall morphology of the films, which in turns is related to the growth mechanism $[8,26]$.

\section{TYPICAL EXAMPLES}

In this article we have concentrated on the structural aspects of multilayered systems. We shall now discuss some typical examples of these systems to understand the merit of the new techniques described in previous sections. An example of x-ray reflectivity study on semiconductor multilayer having device applications is that of an AlAs/AlGaAs Bragg mirror [27]. This consists of 16 thick bilayers grown epitaxially on $\mathrm{GaAs}(001)$. In the extracted EDP (Figure 6) an enhancement of electron density at top surface and an asymmetry of

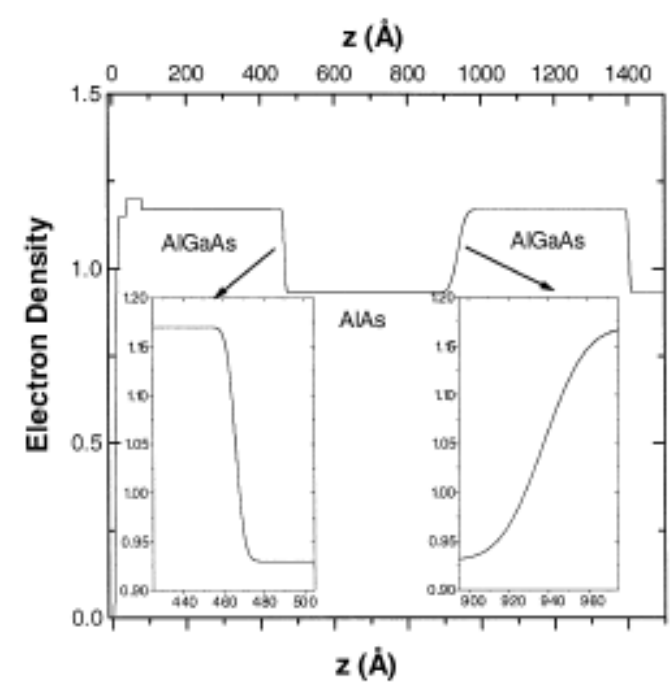

Figure 8. EDP of the first three layers of the Bragg Mirror. Left and right insets show $80 \AA$ of the AlGaAs-on-AlAs and the AlAs-on-AlGaAs interfaces, respectively. 
roughness in the AlAs-on-AlGaAs and AlGaAs-on-AlAs interfaces are observed, the roughness of the former being more than the latter. The layer thicknesses of the $\mathrm{Al}_{0.5} \mathrm{Ga}_{0.5} \mathrm{As}$ and AlAs layers are found to be $465.9 \AA$ and $471.7 \AA$, respectively and apart from the top $80 \AA$ of the film, the electron densities are almost the same as the respective bulk values. It is interesting to note that although the total bilayer thickness obtained from the $\mathrm{x}$-ray analysis has not differed much from the nominal value (937.6 ̊ as compared to $940 \AA$ ), the thickness of AlGaAs has increased by $25.9 \AA$ and AlAs thickness has reduced by almost same amount. This suggests that extra thickness of AlGaAs layer is accommodating the interfacial profile, modeled by an error function. From the FWHM values in a SIMS profile of the multilayered sample, the estimated thicknesses of AlGaAs and AlAs layers are around 465 $\AA$ and $470 \AA$, respectively, which agree with the thicknesses obtained from x-ray studies. Both SIMS and $\mathrm{x}$-ray results indicate the presence of ternary compound, including continuously varying composition of $\mathrm{Al}_{x} \mathrm{Ga}_{1-x} \mathrm{As}_{\text {and }} \mathrm{Al}_{0.5} \mathrm{Ga}_{0.5} \mathrm{As}$ layers in a bilayer.

An example of non-epitaxial semiconductor multilayer is provided by $\mathrm{Ge}-\mathrm{Si}-\mathrm{Ge}$ trilayers grown on $\mathrm{Si}(001)$ single-crystal substrate through ion beam sputtering deposition [28]. The EDP of the trilayer as a function of depth, obtained from x-ray-reflectivity data, reveals an intermixing of $\mathrm{Si}$ and $\mathrm{Ge}$. The x-ray-reflectivity data are analyzed using a scheme based on the distorted-wave Born approximation [17], and the validity of the analysis scheme is checked using simulated data. From the obtained EDP one can estimate the packing fraction of the film as a function of depth (Figure 9(a)). By taking the ratio of the obtained EDP with the packing fraction, an EDP can be obtained which takes into account the porosity of the film (Figure 9(b)). Chemical composition (x) across the depth of the film is then calculated from the EDP values for SixGe1-x (Figure 9(c)). The analyzed results provide information regarding interdiffusion in this system. It can be seen that although the Sion-Ge interface is sharp, a Si0.4Ge0.6 alloy is formed at the Ge-on-Si interface.

Polymer films have been studied extensively using xray and neutron reflectivity $[12,13,29]$ The main prob-
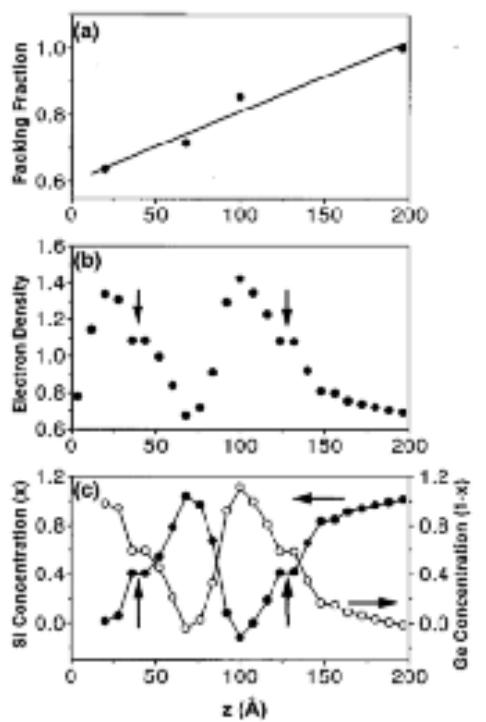

Figure 9: (a) Packing fraction (solid line: linear fit), (b) normalized electron density and (c) concentration of $\mathrm{Si}(\bullet)$ and $\mathrm{Ge}(\mathrm{o})$ as a function of depth for Ge-Si-Ge film.

lem regarding x-ray reflectivity study of polymer multilayer systems is, however, the very low contrast (scattering length density difference) between typical polymers. Fourier methods 
have been developed $[17,30]$ which allow the interfacial parameters to be determined with high accuracy although the difference in the contrast of the two polymers is extremely small. The multilayers, in either pristine or ion-implanted forms find many applications as novel materials, especially in microelectronics and optoelectronics. Particularly interesting examples are polymer quantum wells consisting of self-assembled multilayer films containing alternate layers of conjugated copolymers, and nonconjugated insulating polymers [31]. The photoluminiscence properties of these organic quantum wells can be "tuned" by a proper choice of the conjugated copolymer and the thickness of the insulating layers suggesting a strong spatial confinement. Light emitting diodes based on such self-assembled multilayer films with improved efficiency and stability and with threshold voltage as low as $2.6 \mathrm{~V}$ could be fabricated.

Organic thin films grown by LB techniques [32] are being used to form semiconductor nanoparticles and nanometer sized sheets [33]. However, to control the formation of these useful materials, we need to control the morphology of LB films. GIXS and AFM techniques are being used intensively to understand the enigmatic growth process of LB films [8]. The variation of height-height correlation in these films gives rise to different line shapes in the transverse diffuse scattering profiles. The variation of roughness as a function of length scales in AFM measurement confirmed this x-ray observation (Figure 10). This variation of heightheight correlation ranging from logarithmic to self-affine has been used to predict the growth process of LB films using linear stochastic theory for interface evolution [8]. In-plane structure of LB films has also been investigated in great details using GID [34] and high resolution AFM studies [6] GID has been used to obtain very important results regarding the molecular orientation in the film. AFM studies on the other hand, have provided us valuable information regarding the nature of defects of these films and the melting process in these ultrathin films. X-ray scattering techniques have also been used systematically to understand the defect state [35] and the melting process [9]. The melting process of these ultrathin LB films is an ideal model system to understand two-dimensional melting [10], which is may not have a fixed melting point and believed to be continuous in nature.

Cross-sectional STM and spectroscopy have been used to investigate the atomic-scale structure and electronic properties of $\mathrm{GaN} / \mathrm{GaAs}$ superlattices, which shows that the nitrided layers are laterally inhomogeneous, consisting of groups of atomic-scale defects and larger clusters [36]. Recently, cross-sectional STM is also used to image the interfacial bonding across the nearly lattice-matched, non-common-atom $\mathrm{GaSb} / \mathrm{InAs}$ heterojunction with atomic-scale precision. The method, which takes advantage of the length difference between interfacial and bulk bonds, appears equally applicable to AlSb/InAs and suggests how one might recover the complete structure of either heterojunction from atomic-resolution STM data $[37,38]$. Free-standing wire arrays on a vertically arranged $\mathrm{GaAs} / \mathrm{GaInAs} / \mathrm{GaAs}(001)$ single 

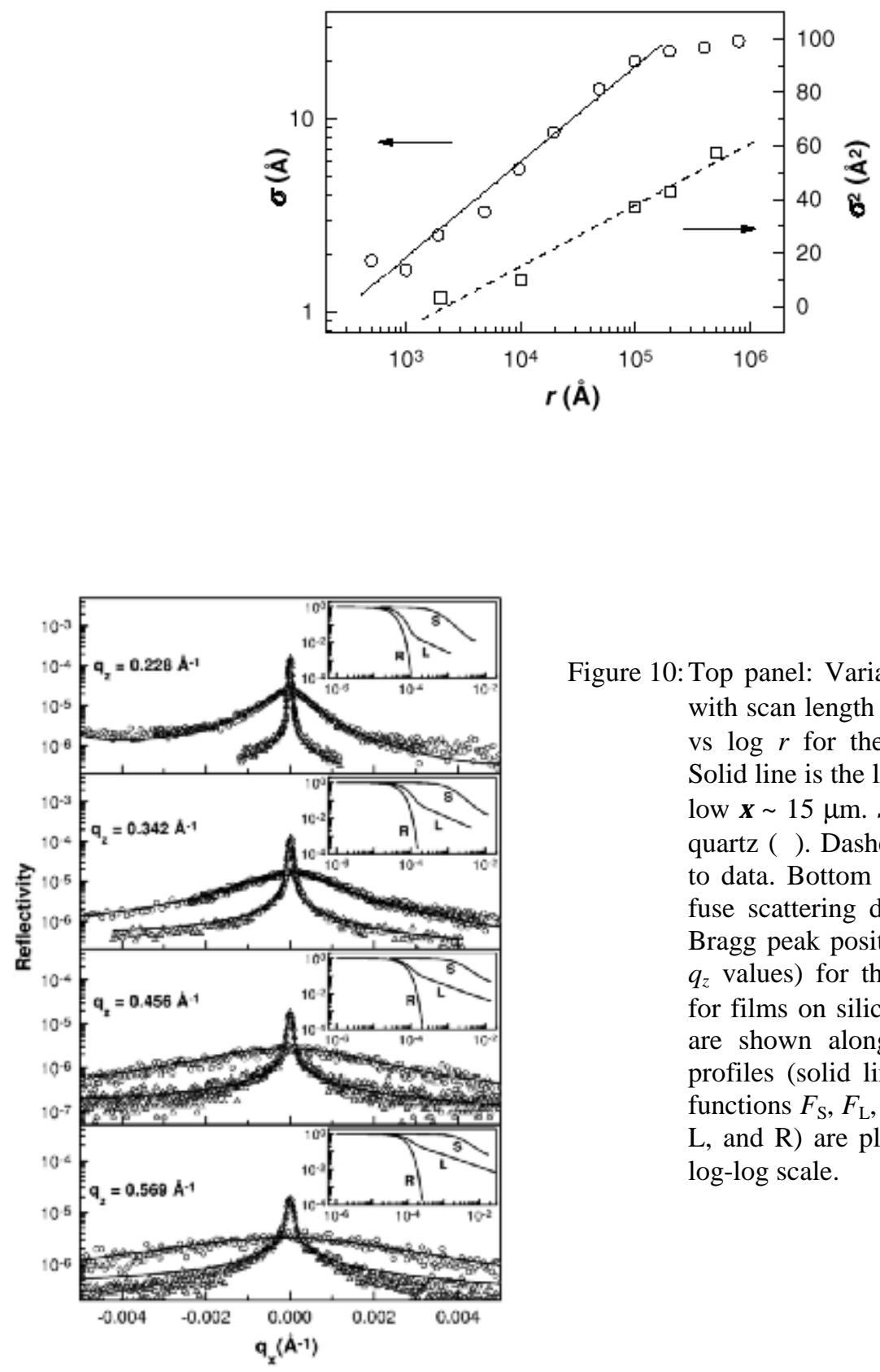

Figure 10: Top panel: Variation of roughness $(\sigma)$ with scan length for the LB films. $\log \sigma$ vs $\log r$ for the film on silicon (O). Solid line is the linear fit to the data below $\xi \sim 15 \mu \mathrm{m} . \sigma^{2}$ vs $\log r$ the film on quartz ( ). Dashed line is the linear fit to data. Bottom panel: Transverse diffuse scattering data at four multilayer Bragg peak positions (indicated by the $q_{z}$ values) for the LB films. The data for films on silicon $(\mathrm{O})$ and quartz $(\Delta)$ are shown along with the calculated profiles (solid lines). In the insets the functions $F_{\mathrm{S}}, F_{\mathrm{L}}$, and $R$ (indicated by $\mathrm{S}$, $\mathrm{L}$, and $\mathrm{R}$ ) are plotted against $q_{x}$ in the $\log$-log scale. 
quantum well structure were characterized by GID based on DWBA [39], and the vertical variation of in-plane strain induced by an $\mathrm{In}_{0.1} \mathrm{Ga}_{0.9}$ As single quantum well embedded in a free-standing wire structure on $\mathrm{GaAs}(001)$ has also been investigated [40]. In the latter, structural parameters of the surface nanostructure were determined with high accuracy and an in-plane lattice displacement within the well with respect to the substrate was found which induces dilative in-plane strain in the GaAs confinement layers decreasing towards the upper free surface and the bulk. Strained-layer superlattices of $\mathrm{Si}_{1-x} \mathrm{Ge}_{x} / \mathrm{Si}$ grown by molecular-beam epitaxy on Si substrates studied by GID [41] revealed a lattice strain relaxation.

\section{FUTURE PROJECTION}

In 1959 Richard Feynman, a celebrated physicist and Nobel Laureate, said, "The principles of physics, as far as I can see, do not speak against the possibility of maneuvering things atom by atom". Recently nano-technology - the technology that can maneuver materials in nanometer length-scales - has promised to deliver these dream materials, known as nanomaterials. In future we may be able to handle materials atom by atom and it is needless to mention that at this level one can make diamond from coal. Even the present nanotechnology has generated many interesting nano-materials and the artificially structured multilayer films having nanometer layer thickness, discussed here, form one group of such materials. Quantum wires, dots and clusters are other examples where atoms are confined within nanometer length-scales in one, two or all three directions.

In these nano-materials most of the atoms are at the surface or interfaces of the structure and interactions of these interfacial atoms dominantly determine the property of these materials. For example in multilayer structures alloys that are not possible to form otherwise, can be formed at the interfaces. It is also possible to form alloys in the entire multilayer stack by heat-treating a multilayer. The composition of the alloy formed by this method can be modified by changing layer-thickness in the original multilayer. The inter-diffusion process responsible for this alloy formation is not fully understood yet.

Ceramic thin films containing nano-particles are interesting materials for the development of new technological devices. Physical properties, namely optical, electrical, magnetic, etc. of such materials are very much different from those of the expected bulk composite materials. The difference is mainly due to the confinement effect of the nanoparticles in the ceramic matrix. This effect arising from the finite size of the nanoparticles is also related to the shape and distribution of the particles in the matrix. It is therefore crucial to understand the morphology before determining the physical properties of such films. By sequential deposition of thin layers of cobalt and alumina, granular multilayers consisting of succes- 
sive planes of nanosized cobalt clusters separated by alumina along the growth direction has been fabricated [42]. Even in ceramic-metal composite thin films prepared by cosputtering techniques, it has been observed that metal nanoparticles are distributed in the ceramic matrix with some preferential layering along the growth direction [43]. The signature of layering in other materials, like polymers and simple liquids, confined within nanometer length-scales has also been observed [17,44].

It is obvious that this field of artificially structured multilayered materials in particular and the field of nano-materials in general will be extremely productive both in the area of fundamental science and technological application. However this research field, like other active frontier research areas, is not as predictable as one wish.

\section{ACKNOWLEDGEMENT}

The authors would like to thank the other faculty members of Surface Physics Division for valuable discussions.

\section{REFERENCES}

1. Current Science (Special Issue on Surface Characterization, Ed.: M.K. Sanyal) 78, 1445-1531 (2000).

2. W. Hansen, J. P. Kotthaus, and U. Merkt, Semicond. Semimetals 35, 279 (1992).

3. F. J. Himpsel, J. E. Ortega, G. J. Mankey, and R. F. Willis, Adv. Phys. 47, 511 (1998).

4. M. A. M. Gijs and G. E. W. Bauer, Adv. Phys. 46, 285 (1997).

5. T. P. A. Hase, I. Pape, B. K. Tanner, H. Dürr, E. Dudznik, G. van der Laan, C. H. Marrows, and B. J. Hickey, Phys. Rev. B 61, R3792 (2000) and references therein.

6. D. K. Schwartz, Surf. Sci. Rep. 27, 241 (1997).

7. G. L. Gaines, Jr., Insoluble Monolayers at the Gas-Liquid Inter-face (Wiley, New York, 1966)

8. J. K. Basu, S. Hazra and M. K. Sanyal, Phys. Rev. Lett. 82, 4675 (1999).

9. J. K. Basu, M. K. Sanyal, M. Mukherjee and S. Banerjee, Phys. Rev. B 62, 11109 (2000).

10. J. M. Kosterlitz and D. J. Thouless, J. Phys. C 6, 1181 (1973).

11. Physica B 283 (2000) and earlier SXNS Proceedings.

12. M. Tolan, X-ray Scattering from Soft-Matter Thin Films, Springer Tracts in Modern Physics, (Springer, Berlin 1999).

13. J. Daillant and A. Gibaud Eds., X-ray and Neutron Reflectivity, Principles and Applications, Lecture Notes in Physics (Springer, Berlin 1999).

14. S. K. Sinha, E. B. Sirota, S. Garoff, and H. B. Stanley, Phys. Rev.B 38, 2297 (1988).

15. I. K. Robinson and D. J. Tweet, Rep. Prog. Phys. 55, 599 (1992).

16. L. G. Parratt, Phys. Rev. 95, 359 (1954).

17. M. K. Sanyal, J. K. Basu, A. Datta and S. Banerjee, Europhys. Lett. 36, 265 (1996)

18. A. van der Lee, Eur. Phys. J. B 13, 755 (2000). 
19. M. K. Sanyal, S. Hazra, J. K. Basu, and A. Datta, Phys. Rev. B 58, R4258 (1998).

20. S. K. Sinha, Current Opinions in Solid State and Materials Science 1, 645 (1996).

21. S. K. Sinha, M. K. Sanyal, S. K. Satija, C. F. Majkrzak, D. A. Neumann, H. Homma, S. Szpala, A. Gibaud, and H. Markoc, Physica B 198, 72 (1994).

22. M. K. Sanyal, S. K. Sinha, A. Gibaud, S. K. Satija, C. F. Majkrzak, and H. Homma, Mater. Res. Soc. Symp. Proc. 237, 393 (1992).

23. G. H. Vineyard, Phys. Rev. B 26, 4146 (1982).

24. A. Datta, J. Kmetko, C.-J. Yu, A. G. Richter, K.-S. Chung, J.-M. Bai, and P. Dutta, J. Phys. Chem. B, 104, 5797 (2000).

25. D. Sarid, Scanning Force Microscopy (Oxford University Press, New York, 1991); C. J. Chen, Introduction to Scanning Tunneling Microscopy (Oxford University Press, New York, 1993).

26. S. Kundu, S. Hazra, S. Banerjee, M. K. Sanyal, S. K. Mandal, S. Chaudhuri and A. K. Pal; J. Phys. D 31, L73 (1998).

27. M. K. Sanyal, A. Datta, A.K. Srivatsava, B. M. Arora, S. Banerjee, P. Chakraborty, F. Caccavale, O. Sakata, and H. Hashizume, Appl. Surf. Sci. 133, 98 (1998).

28. S. Banerjee, M. K. Sanyal, A. Datta, S. Kanakaraju and S. Mohan, Phys. Rev. B 54, 16377 (1996).

29. T. P. Russell, Mater. Sci. Rep. 5, 171 (1990).

30. O. H. Seeck, I. D. Kaendler, M. Tolan, K. Shin, M. H. Rafailovich, J. Sokolov and R. Kolb, Appl. Phys. Lett. 76, 2713 (2000).

31. H. Hong, M. Tarabia, H. Chayet, D. Davidov, E. Z. Faraggi, Y. Avny, R. Neumann and S. Kirstein, J. Appl. Phys. 79, 3082 (1996).

32. A. Datta, M.K. Sanyal, A. Dhanabalan, and S.S. Major, J. Phys. Chem. 101, 9280 (1997).

33. J. K. Basu and M.K. Sanyal, Phys. Rev. Lett. 79, 4617 (1997).

34. A. Malik, M. K. Durbin, A. G. Richter, K. G. Huang and P. Dutta, Phys. Rev. B 52, 11654 (1995).

35. R. Stömmer and U. Pietsch, J. Phys. D 29, 3161 (1996).

36. R. S. Goldman, R. M. Feenstra, B. G. Briner, M. L. O'Steen and R. J. Hauenstein, Appl. Phys. Lett. 69, 3698 (1996).

37. J. Steinshnider, M. Weimer, R. Kaspi and G. W. Turner, Phys. Rev. Lett. 85, 2953 (2000).

38. J. Yu, J. L. Cao, Y. Namba and Y. Y. Ma, J. Vac. Sci. Tech. B 14, 42 (1996).

39. K. Paschke, T. Geue, T. A. Barberka, A. Bolm, U. Pietsch, M. Rösch, E. Batke, F. Faller, K. Kerkel, J. Oshinowo, and A. Forchel, Appl. Phys. Lett. 70, 1031 (1997).

40. A. Ulyanenkov, T. Baumbach, N. Darowski, U. Pietsch, K. H. Wang, A. Forchel and T. Wiebach, J. Appl. Phys. 85, 1524 (1999).

41. Z. H. Mai, J. T.- Ouyang, S. F. Cui, J. H. Li, C. Y. Wang, and C. R. Li, J. Appl. Phys. 72, 3474 (1992).

42. D. Babonneau, F. Petroff, J. -L. Maurice, F. Fettar, A. Vaures and A. Naudon, Appl. Phys. Lett. 76, 2892 (2000).

43. S. Hazra, A. Gibaud, A. Desert, C. Sella and A. Naudon; Physica B 283, 97 (2000).

44. C.-J. Yu, A.G. Richter, A. Datta, M. K. Durbin and P. Dutta, Phys. Rev. Lett. 82, 2326 (1999). 